\title{
Adiabatic-Nonadiabatic Transition in Warm Long-Range Interacting Systems: The Transport of Intense Inhomogeneous Beams
}

\author{
Everton G. Souza, ${ }^{1,2, *}$ Antonio Endler, ${ }^{1, \dagger}$ Felipe B. Rizzato, ${ }^{1,+}$ and Renato Pakter ${ }^{1, \S}$ \\ ${ }^{1}$ Instituto de Física, Universidade Federal do Rio Grande do Sul, \\ Caixa Postal 15051, 91501-970, Porto Alegre, Rio Grande do Sul, Brazil \\ ${ }^{2}$ CERN, Accelerators and Beam Physics Group, CH-1211 Geneva, Switzerland
}

(Received 27 April 2012; published 17 August 2012)

\begin{abstract}
We investigate the role of the temperature in the onset of singularities and the consequent breakdown in a macroscopic fluid model for long-range interacting systems. In particular, we consider an adiabatic fluid description for the transport of intense inhomogeneous charged particle beams. We find that there exists a critical temperature below which the fluid model always develops a singularity and breaks down as the system evolves. As the critical temperature is approached, however, the time for the occurrence of the singularity diverges. Therefore, the critical temperature separates two distinct dynamical phases: a nonadiabatic transport at lower temperatures and a completely adiabatic evolution at higher temperatures. These findings are verified with the aid of self-consistent $N$-particle simulations.
\end{abstract}

DOI: 10.1103/PhysRevLett.109.075003

PACS numbers: 52.59. $-\mathrm{f}, 51.30 .+\mathrm{i}, 52.27 . \mathrm{Jt}$

For long-range self-interacting systems, it is generally very difficult to obtain a fully kinetic description of the dynamics. This is the case in plasmas, charged particle beams, and self-gravitating systems among others, where the collision duration time diverges in the thermodynamic limit [1-5]. A largely used tool to overcome this difficulty is the employment of macroscopic fluid models. In contrast to the kinetic description that requires the knowledge of the evolution of the distribution function in the full phase space, the fluid description is simpler because it is based on local macroscopic variables obtained by averaging over the momentum space. Moreover, because the fluid variables consist of readily understood macroscopic quantities, the physical interpretation of the phenomena under investigation is generally more direct. Nevertheless, except for very specific cases, the fluid description leads to an infinite hierarchy of equations which, in practice, have to be truncated to be analyzed. The truncation is obtained by assuming a certain characteristic for the system dynamics which is expressed by an equation of state. Perhaps, the simplest used approximation is the cold fluid, which completely neglects thermal effects by assuming a vanishing temperature [6-12]. Other examples of largely used equations of state are isothermal [13-17] and adiabatic $[18,19]$. At any rate, the validity of the fluid description resides not only on the choice of equation of state but also on the fact that the infinite hierarchy has to be convergent; i.e., the macroscopic fluid variables may not present singularities. In the case of cold fluids, a wellknown cause of singularities is the onset of the so-called wave breaking where the fluid description looses its validity due to a divergence in the particles density at a certain position and time. This phenomenon is associated with a filamentation in the phase space and may have relevant consequences such as temperature increase, energy redistribution, and particle acceleration, depending on the system.

In this Letter, we investigate the role of the temperature in the onset of singularities in the macroscopic quantities and the consequent breakdown of the fluid description. In particular, we consider the transport of intense inhomogeneous charged particle beams along a uniform focusing magnetic field channel. The inhomogeneity is taken into account because it is virtually impossible to launch a perfectly homogeneous beam in real devices [20,21]. In the cold beam limit, this system was shown to present wave breaking which is responsible for particle ejection from the beam core and has a very deleterious effect in the transport $[11,12]$. Here, we develop a Lagrangian fluid model for the beam evolution that incorporates thermal effects using an adiabatic approximation. It is found that any finite temperature is sufficient to prevent the occurrence of the divergence in the beam density and the associated wave breaking. Nevertheless, if the temperature is below a certain threshold we identify a different type of singularity that is responsible for the breakdown of the fluid description, namely, the formation of colliding layers where beam regions with significantly different densities bump into each other leading to a singular growth of the pressure gradient. As the threshold temperature is approached, however, the time for the occurrence of the singularity diverges and is found to be absent above it. In other words, the fluid model predicts the existence of a critical temperature that separates two different dynamical phases: a nonadiabatic transport at lower temperatures and a completely adiabatic evolution at higher temperatures. These findings are verified with the aid of self-consistent $N$-particle simulations.

We consider a continuous, stationary, axisymmetric beam that propagates along a $z$ axis with a constant axial velocity $v_{0}[22,23]$. The beam is focused by a uniform 
magnetic field of magnitude $B$ that is directed along $z$. For a given beam distribution at $z=0$, our aim is to determine how the beam evolves as a function of the axial coordinate $z=v_{0} t$, which plays the role of time in our system. Since the number of constituent particles is very large, the beam dynamics is dictated by collective effects and interparticle collisions are negligible. Using Lagrangian coordinates [24] and the paraxial approximation [22], the dynamics of the transverse radial position $r$ of a certain cylindrical beam element that started at $r_{0}$ at $z=0$ is governed by

$$
\frac{d^{2} r}{d z^{2}}=-\kappa r+\frac{Q\left(r_{0}\right)}{r}-\frac{1}{\rho(r)} \frac{\partial P}{\partial r} .
$$

The first term on the right-hand side of Eq. (1) corresponds to the focusing force imposed by the external magnetic field, where $\kappa \equiv\left(q B / 2 \gamma m \beta c^{2}\right)^{2}$ is the focusing parameter, $\gamma=\left(1-\beta^{2}\right)^{-1 / 2}$ is the relativistic mass factor, $\beta=v_{z} / c, c$ is the speed of light in vacuo, and $q$ and $m$ denote the beam particle charge and mass, respectively. The second term gives the space charge forces and is readily obtained by taking advantage of the axisymmetry of the beam and using Gauss law, where $Q(r)=K N(r) / N_{t}$ is a measure of the charge contained up to the fluid element position at $r, N_{t}$ is the total number of beam particles per unit axial length, $N(r)$ is the number of particles up to $r$, and $K=2 N_{t} q^{2} / \gamma^{3} m \beta^{2} c^{2}$ is the beam perveance. Note that a necessary condition for the validity of the fluid description is that different fluid elements do not overtake each other. As a consequence, $Q(r)$ is invariant and may be evaluated as the initial value $Q\left(r_{0}\right)$. Likewise, imposing the conservation of charge between two neighboring layers located at $r$ and $r+d r$, we can write

$$
\rho(r, z)=\frac{r_{0}}{r C(r)} \rho_{0}\left(r_{0}\right)
$$

which determines the evolution of the beam particle density $\rho(r, z)$ as a function of the initial density at $\rho_{0}\left(r_{0}\right)$, where $C(r)=d r / d r_{0}$ is the so-called fluid compressibility. Finally, the third term on the right-hand side of Eq. (1) introduces the thermal effects, where $P$ is the transverse fluid pressure. To determine a pressure profile, we assume that the beam transport is adiabatic; i.e., the quantity $P / \rho^{2}$ of a given fluid element is conserved along its trajectory [19]. We also assume that the beam is injected at $z=0$ with a uniform temperature $T_{0}$, such that $P_{0}\left(r_{0}\right)=$ $\rho_{0}\left(r_{0}\right) T_{0}$ at injection. Thus, using Eq. (2) the pressure profile takes the form

$$
P\left(r, r_{0}\right)=\frac{\rho_{0}\left(r_{0}\right) r_{0}^{2} T_{0}}{r^{2} C^{2}(r)} .
$$

It is worth noting that in order to correctly evaluate the pressure gradient in Eq. (1) one has to explicitly take into account the variations of $r_{0}$ with respect to $r$ by using $d r_{0} / d r=1 / C(r)$.
To numerically solve the fluid model, we consider $N_{f}$ fluid elements whose initial conditions $r_{0}$ are uniformly distributed from zero to an initial maximum radius $r_{b 0}$. The position $r\left(r_{0}, z\right)$ of each fluid element is then obtained by simultaneously integrating the set of $N_{f}$ ordinary differential equations of motion (1) for each $r_{0}$. The derivatives with respect to $r$ and $r_{0}$ are approximated by finite differences between neighboring elements. In the simulations, we consider beams whose initial profiles are parabolic,

$$
\rho_{0}\left(r_{0}\right)=\frac{K}{\pi r_{b 0}^{2}}\left[1+\chi\left(\frac{2 r_{0}^{2}}{r_{b 0}^{2}}-1\right)\right]
$$

for $r_{0}<r_{b 0}$, where $-1 \leq \chi \leq+1$ is a parameter that measures the degree of inhomogeneity. We focus on matched beams with $r_{b 0}=(K / \kappa)^{1 / 2}$ which corresponds to a vanishing small force acting upon the outermost particle [11]. This condition prevents the onset of azimuthal instabilities that could break the axisymmetry of the beam [25]. For $\chi=0$ the beam is homogeneous, which corresponds to a quasiequilibrium condition and a nearly stationary evolution.

As mentioned before, a necessary condition for the validity of the fluid model is that neighboring fluid elements do not overtake each other. In fact, if at a certain instant two fluid elements that started at different locations occupy the same position then the compressibility $C$ vanishes at that point. According to Eq. (2), this leads to a singular build up of the density and to a wave breaking. This phenomenon was shown to always occurs in the transport of initially inhomogeneous $(\chi \neq 0)$ cold beams and is responsible for particle ejection and consequent degradation of the beam quality $[6,11]$. In the presence of thermal effects, however, the pressure gradient term in Eq. (1) is sufficient to inhibit such a process, and $C$ is found to never vanish. Nevertheless, we have identified that another event that breaks down the fluid description may occur, namely, the formation of colliding layers where beam regions with significantly different densities bump into each other. This phenomenon is characterized by the onset of a discontinuity in the $C\left(r_{0}\right)$ profile, as shown by the dots in Fig. 1 at $r_{0} \approx 0.5$; to the left (smaller $r_{0}$ ) of the discontinuity there is a higher density layer that is coming across a lower density layer to the right. Because the derivative of the compressibility diverges at this point, it corresponds to a singular growth of the pressure gradient in Eq. (1). Similar to the wave breaking, the occurrence of colliding density layers may as well be responsible for conversion of energy from macroscopic fluid modes into microscopic kinetic activity and consequent degradation of the beam. Therefore, it becomes relevant to identify when this effect occurs.

In order to determine this, we ran the fluid simulation for different system parameters and determined if and at which time $z^{*}$ the fluid description lost validity. The runs were performed for times much longer than the wave breaking 


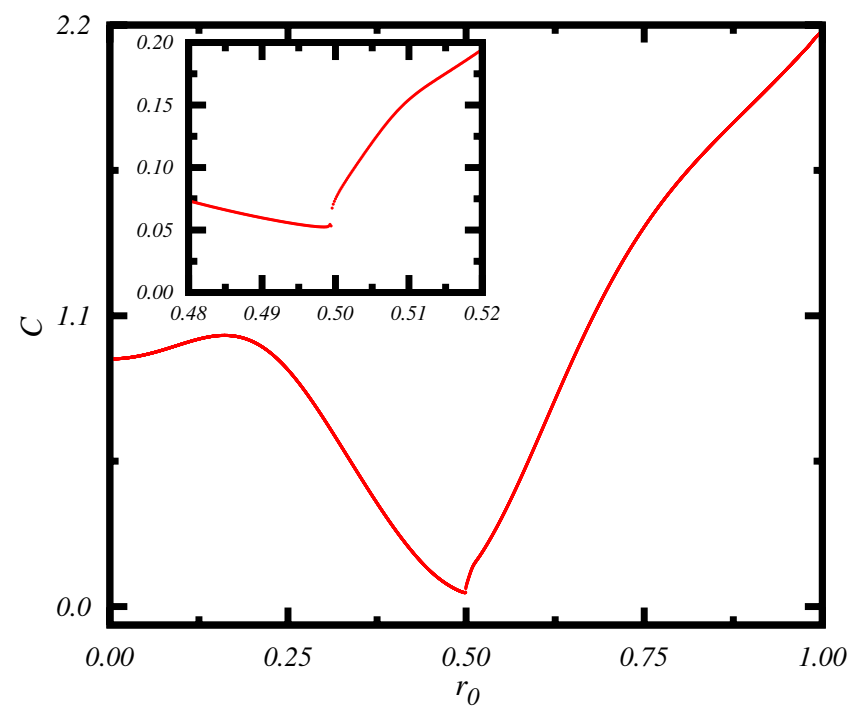

FIG. 1 (color online). Compressibility $C$ as a function of the initial position $r_{0}$ showing the onset of a discontinuity close to $r_{0}=0.5$. Because the density is inversely proportional to $C$ [see Eq. (2)], the discontinuity indicates the formation of colliding layers where a higher density segment of the beam to the left of the discontinuity is bumping into a lower density layer to the right. This phenomena leads to a breakdown of the fluid description. The inset shows a zoom of $C\left(r_{0}\right)$ near the discontinuity. This curve was obtained for $T_{0}=10^{-6}, \chi=0.6$, and $z \approx z_{0}^{*} \approx$ 125 , where $z_{0}^{*}$ is the time for the wave breaking in the cold beam limit.

time for $T_{0}=0$ that we shall denote as $z_{0}^{*}$. The results are represented in color code in the $\chi$ vs $T_{0}$ parameter space of Fig. 2. The gray scale points refer to the parameter values for which $z^{*}<z_{0}^{*}$, whereas the colored points refer to $z^{*}>z_{0}^{*}$. For low temperatures, we see a large black area that indicates that $z^{*} \approx z_{0}^{*}$. Therefore, despite the fact that the phenomena that leads to the breakdown of the fluid model is intrinsically different for vanishing and finite temperatures, the time for their occurrences is very similar at low temperatures. On the other hand, for the higher temperatures in Fig. 2 there is a large yellow area that corresponds to the complete absence of colliding density layers along the runs. In between these areas there is a thin transition region (in blue) where $z^{*}$ changes very rapidly. This region defines a threshold temperature $T_{0 c}(\chi)$ above which the fluid description becomes valid throughout the transport. I.e., a small variation in the temperature completely changes the system dynamics: while for a temperature slightly lower than $T_{0 c}$ the fluid breaks down on the same time scales as of a cold beam, for a temperature slightly higher than $T_{0 c}$ the beam flow becomes mostly adiabatic.

To further explore the significance of the break down of the fluid description and how it affects beam quality, we have performed fully kinetic self-consistent $N$-particle simulations. Details of the simulation method can be found

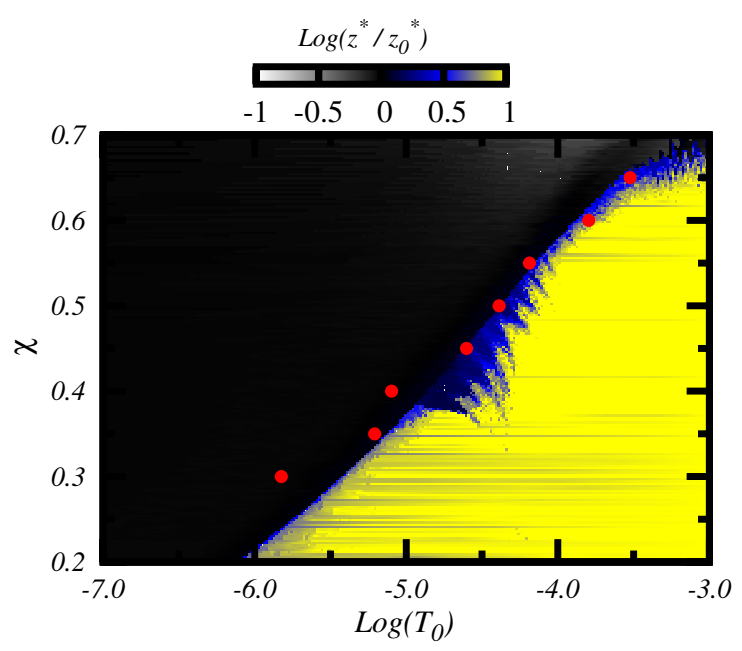

FIG. 2 (color online). Color coded map of the time for the break up of the fluid description in the $T_{0}$ vs $\chi$ parameter space. The large black area indicates that the break up time is of the same order of the cold one $\left(z^{*} \approx z_{0}^{*}\right)$ at low temperatures. The thin blue (lighter) region defines a threshold temperature $T_{0 c}(\chi)$ at which $z^{*}$ changes very rapidly and above which the fluid description becomes valid throughout the simulation (light yellow area). The red dots correspond to the threshold temperature $T_{0 c}$ obtained from the $N$-particle self-consistent simulations for different values of $\chi$ (see Fig. 4). In this figures we concentrate on values of $\chi$ that lead to a slow wave breaking in the cold beam limit [12].

in Ref. [26]. In the simulations, we launch $N=20000$ macroparticles according to the parabolic beam profile of Eq. (4) with prescribed $\chi$ and $r_{b 0}$, and with velocities uniformly distributed up to a maximum speed $v_{m}$, such that the initial temperature is $T_{0}=v_{m}^{2} / 2$. Generally, the beam quality is quantified using the emittance given by $\varepsilon^{2}(z)=4\left(\left\langle r^{2}\right\rangle\left\langle\mathrm{v}^{2}\right\rangle-\langle\mathbf{r} \cdot \mathbf{v}\rangle^{2}\right)$, where $\mathbf{r}$ and $\mathbf{v}$ are the position and velocity vectors of the beam particles, respectively, and $\langle\cdots\rangle$ stands for average over all particles. In the case of inhomogeneous beams, the charge redistribution inside the beam causes fast plasma oscillations which largely affect the evolution of $\varepsilon(z)$ [11]. Therefore, it is convenient to rewrite the emittance squared as the exact sum of two terms, $\varepsilon^{2}=\varepsilon_{d}^{2}+\varepsilon_{\text {the }}^{2}$, where we explicitly separate the contributions from the (macroscopic) plasma oscillations in a directed emittance $\varepsilon_{d}^{2}=4\left[\left\langle r^{2}\right\rangle\left\langle\bar{v}^{2}\right\rangle-\langle\mathbf{r} \cdot \overline{\mathbf{v}}\rangle^{2}\right]$ and from the microscopic kinetic activity in a thermal emittance

$$
\varepsilon_{\text {the }}^{2}=4\left[\left\langle r^{2}\right\rangle\left\langle(\mathbf{v}-\overline{\mathbf{v}})^{2}\right\rangle\right],
$$

where $\overline{\mathbf{v}}(\mathbf{r})$ is the macroscopic fluid velocity obtained by averaging over the particles in the vicinity of the position $\mathbf{r}$. Because the thermal emittance growth is the one associated to the irreversible entropy increase as particles are ejected from the core to form halos, we focus on its evolution to determine beam quality. In Fig. 3, we present the evolution of $\varepsilon_{\text {the }}(z)$ for $\chi=0.6$ and two different temperatures which 


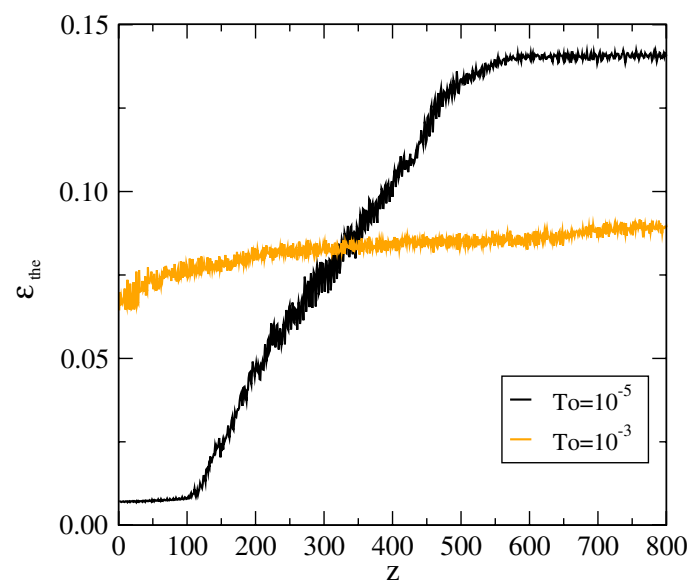

FIG. 3 (color online). Evolution of the thermal emittance defined in Eq. (5) for temperatures below and above the critical temperature $T_{0 c} \approx 10^{-4}$ (see Fig. 2). For $T_{0}=10^{-5}<T_{0 c}, \varepsilon_{\text {the }}$ has virtually no growth up to $z \approx z_{0}^{*}=122$ where it starts to grow very fast (dark curve). On the other hand, for $T_{0}=10^{-3}>T_{0 c}$ the simulation is nearly adiabatic with just a slow increase in $\varepsilon_{\text {the }}$ (light curve).

are respectively below and above the critical temperature $T_{0 c} \approx 10^{-4}$. In the former case, $\varepsilon_{\text {the }}$ has virtually no growth up to $z \approx z_{0}^{*}=122$ where it starts to grow very fast. This can be explained with the aid of the adiabatic fluid model that predicts the occurrence of colliding density layers at $z \approx z_{0}^{*}$ for this set of parameters. On the other hand, for $T_{0}>T_{0 c}$ the fluid theory predicts no singularities, and correspondingly, the transport seen in the simulation is nearly adiabatic with just a slow increase in $\varepsilon_{\text {the }}$. In Fig. 4, we show the thermal emittance variation obtained as the difference between the final and the initial thermal emittance over a certain fixed distance $z$ for $\chi=0.6$ and various values of $T_{0}$. In agreement with the predictions from the fluid model, it is clear that there is a critical value of the temperature $T_{0 c}$ above which the beam transport becomes nearly adiabatic with just a minor increase in the thermal emittance. Similar analyses have also been done for different values of $\chi$, in order to determine the corresponding $T_{0 c}$. The results are presented by the circles in Fig. 2. Again, good agreement is found between the fluid theory and the $\mathrm{N}$-particle simulations.

In conclusion, we have investigated the role of the temperature in the onset of singularities and the consequent breakdown in a macroscopic fluid model. In particular, we considered the transport of intense inhomogeneous charged particle beams which are known to present a wave breaking in the cold limit. Using an adiabatic fluid model to describe the beam transport, we found that any finite temperature is sufficient to prevent the occurrence of the wave breaking. Nevertheless, if the temperature is below a certain threshold we identified the occurrence of a different type of singularity that is responsible for the breakdown of the fluid description, namely, the formation

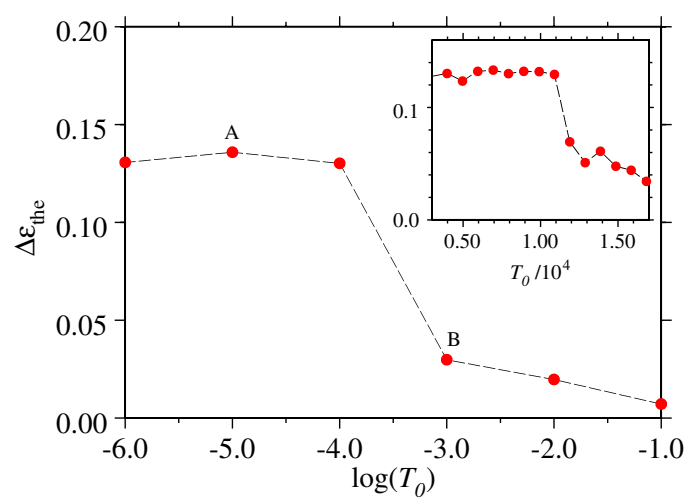

FIG. 4 (color online). Thermal emittance variation $\Delta \varepsilon_{\text {the }}$ as a function of the temperature $T_{0}$ for $\chi=0.6 . \Delta \varepsilon_{\text {the }}$ is computed as the difference between the final and the initial thermal emittance over a certain fixed distance of propagation. Points $A$ and $B$ correspond to the values of $T_{0}$ used in Fig. 3. A critical temperature $T_{0 c} \approx 10^{-4}$ is found above which the beam transport becomes nearly adiabatic with just a minor increase in the thermal emittance. The inset shows a zoom in the vicinity of $T_{0 c}$ in a linear plot in $T_{0}$.

of colliding layers where beam regions with significantly different densities bump into each other leading to a singular growth of the pressure gradient. As the threshold temperature is approached, however, the time for the occurrence of the singularity diverges and is absent above it. Therefore, depending on the parameters of the system, we find the existence of a critical temperature that separates two different dynamical phases: a nonadiabatic transport at lower temperatures and a completely adiabatic evolution at higher temperatures. These findings were verified with the aid of self-consistent $N$-particle simulations.

This work was partially supported by $\mathrm{CNPq}$ and FAPERGS, Brazil, and by the US-AFOSR under Grant No. FA9550-09-1-0283.

*evertongs@gmail.com.br
${ }^{\dagger}$ aendler@if.ufrgs.br
${ }^{\ddagger}$ rizzato@if.ufrgs.br
${ }^{\S}$ pakter@if.ufrgs.br

[1] W. Braun and K. Hepp, Commun. Math. Phys. 56, 101 (1977).

[2] Y. Levin, R. Pakter, and T. N. Teles, Phys. Rev. Lett. 100, 040604 (2008).

[3] A. Campa, T. Dauxois, and S. Ruffo, Phys. Rep. 480, 57 (2009).

[4] T. N. Teles, Y. Levin, R. Pakter, and F. B. Rizzato, J. Stat. Mech. (2010) P05007.

[5] R. Pakter and Y. Levin, Phys. Rev. Lett. 106, 200603 (2011).

[6] J. M. Dawson, Phys. Rev. 113, 383 (1959).

[7] J. Swegle and E. Ott, Phys. Rev. Lett. 46, 929 (1981).

[8] L. Serafini and J. B. Rosenzweig, Phys. Rev. E 55, 7565 (1997). 
[9] R. Pakter and C. Chen, Phys. Rev. E 62, 2789 (2000).

[10] N. Kjærgaard and M. Drewsen, Phys. Rev. Lett. 91, 095002 (2003).

[11] F. B. Rizzato, R. Pakter, and Y. Levin, Phys. Plasmas 14, 110701 (2007).

[12] E. G. Souza, A. Endler, R. Pakter, F. B. Rizzato, and R. P. Nunes, Appl. Phys. Lett. 96, 141503 (2010).

[13] B. Semelin, N. Sanchez, and H. J. de Vega, Phys. Rev. D 63, 084005 (2001).

[14] C. M. Pooley and J. M. Yeomans, Phys. Rev. Lett. 93, 118001 (2004).

[15] G. H. Goedecke, B. T. Davis, C. Chen, and C. V. Baker, Phys. Plasmas 12, 113104 (2005).

[16] S. Galtier and S. Banerjee, Phys. Rev. Lett. 107, 134501 (2011).

[17] E. G. Altmann and A. Endler, Phys. Rev. Lett. 105, 244102 (2010).
[18] A. Ori and T. Piran, Phys. Rev. Lett. 59, 2137 (1987).

[19] S. M. Lund and R. C. Davidson, Phys. Plasmas 5, 3028 (1998).

[20] S. S. Yu, S. Eylon, E. Henestrosza, D. P. Grote, in Proceedings of the Workshop on Space Charge Dominated Beams and Applications of High Brightness Beams, 1995 (AIP, New York, 1996), p. 134.

[21] S. Bernal, R. A. Kishek, M. Reiser, and I. Haber, Phys. Rev. Lett. 82, 4002 (1999).

[22] M. Reiser, Theory and Design of Charged Particle Beams (John Wiley, New York, 1994).

[23] R. Pakter and F. B. Rizzato, Phys. Rev. Lett. 87, 044801 (2001).

[24] P. J. Morrison, Rev. Mod. Phys. 70, 467 (1998).

[25] W. Simeoni, Jr., F. B. Rizzato, and R. Pakter, Phys. Plasmas 13, 063104 (2006).

[26] H. Okamoto and M. Ikegami, Phys. Rev. E 55, 4694 (1997). 